\title{
ERP SOURCE RECONSTRUCTION BY USING PARTICLE SWARM OPTIMIZATION
}

\author{
Y.K. Alp, O. Arikan \\ Bilkent University \\ Electrical and Electronics Engineering \\ Ankara, Turkey
}

\author{
S. Karakas* \\ Hacettepe University \\ Conjuitive Psychophysiology Research Unit \\ Ankara, Turkey
}

\begin{abstract}
Localization of the sources of Event Related Potentials (ERP) is a challenging inverse problem, especially to resolve sources of neural activity occurring simultaneously. By using an effective dipole source model, we propose a new technique for accurate source localization of ERP signals. The parameters of the dipole ERP sources are optimally chosen by using Particle Swarm Optimization technique. Obtained results on synthetic data sets show that proposed method well localizes the dipoles on their actual locations. On real data sets, the fit error between the actual and reconstructed data is successfully reduced to noise level by localizing a few dipoles in the brain.
\end{abstract}

Index Terms - particle swarm optimization(PSO), analysis of neural activity, ERP source localization.

\section{INTRODUCTION}

Event Related Potentials (ERPs) are observable results of neural activity of the brain to a controlled stimulus, providing very important clues on the cognitive processes. They are also useful to identify neurological problems such as Attention Deficit Hyperactivity Disorders [1]. The ERP signals are recorded by using a set of electrodes, or sensors, that are closely spaced on the skull. A set of audio or visual stimuli are used to generate neural activity resulting in spatially localized currents that can be modeled by an effective current dipole source [2]. There are various signal processing techniques that are developed for extraction of different types of information from the recorded ERP channels. One of the most challenging problems in the ERP signal processing is the localization of individual ERP sources [3].

In this paper, we propose a new signal processing technique developed for the ERP source localization. Unlike existing alternatives, Particle Swarm Optimization technique is used for obtaining global minima of a fit error between the synthetic and actual ERP channels. As a result of the optimization, a fixed location and orientation for each dipole source are obtained that will remain constant in the time interval of interest. Hence, unlike the existing approaches where

\footnotetext{
*The third author provided the real EEG data used in this work.
}

dipole source locations are determined for every time sample, the new method is free from the traveling dipole artifacts [4].

After a short review on a commonly used forward model for the ERP channels, we present the formulation of the optimization problem and its solution based on Particle Swarm Optimization technique. Performance of the proposed source reconstruction technique is investigated over both synthetic and real ERP channel data.

\section{FORWARD MODEL FOR ERP MEASUREMENTS}

The solution for the source reconstruction for the ERP channel data requires a forward model where the ERP measurements are related to the current sources generated by the neural activity of the brain. There are different types of commonly used forward models for this purpose [5]. Two of them make use of finite element model and boundary element model for the head, and relate the measurements to the individual current components defined on a high resolution grid. This type of forward models are useful to reconstruct current density reconstructions. However, due to the size of the computational grid, the inverse problem becomes highly illconditioned, and only regularized estimates of the current densities can be estimated, which tends to have artificially smooth and not so localized characteristics [6]. Since an important feature for a desired reconstruction is having localized ERP sources, these forward models are not appropriate for our purpose. Therefore, we use an other alternative model for the ERP sources where few localized dipole sources are utilized. Before getting into the details of the dipole forward model it is important to note that even if the neural activity is a result of localized current sources, it can be accurately modeled by using its effective dipole model.

In the effective dipole based forward model of the ERP measurements, there are two important components: the conductivity model for the head, and the source model of the neural activity. Typically the conductivity model for the head is chosen as a multishell model where the head is represented as three concentric spheres corresponding to brain, skull and the scalp, with their respective conductivities [7]. The center and the scalp radius of the concentric spheres can 
be determined from a best sphere fit to the known electrode locations on the surface of the scalp. If available, based on a structural MR scan, the scalp and skull thickness values can be determined. Otherwise, age related average values can be assumed. For the conductivity values of different tissues, commonly used tabulated values can be used [8]. For the source model of the neural activity, a regional dipole model with fixed locations and orientations but time varying modulations is used [2]. Specifically, for $N_{s}$ regional dipoles having locations $\mathbf{r}_{j}=\left\{r_{x j}, r_{y j}, r_{z j}\right\}$, and moments $\mathbf{m}_{j}=\left\{\cos \theta_{j} \cos \phi_{j}, \cos \theta_{j} \sin \phi_{j}, \sin \phi_{j}\right\}, j=1,2, . ., N_{s}$ where $\theta_{j}$ and $\phi_{j}$ are polar angles defining the orientation of the dipole, the potential measured at the $i^{t h}$ electrode position $\mathbf{s}_{i}=\left\{s_{x i}, s_{y i}, s_{z i}\right\}$ can be modeled as:

$$
v\left(\mathbf{s}_{i}, t\right)=\sum_{j=1}^{N_{s}} a_{j}(t) \psi\left(\mathbf{s}_{i}, \mathbf{r}_{j}, \mathbf{m}_{j}\right)
$$

where $a_{j}(t)$ represents the time-varying modulation of the $i^{t h}$ dipole and $\psi\left(\mathbf{s}_{i}, \mathbf{r}_{j}, \mathbf{m}_{j}\right)$ is potential measured at the electrode position $\mathbf{s}_{i}$ generated by the unit energy dipole located at $\mathbf{r}_{j}$ and having moment $\mathbf{m}_{j}$. According to the head model, $\psi\left(\mathbf{s}_{i}, \mathbf{r}_{j}, \mathbf{m}_{j}\right)$ is computed by the following formula given in [7]:

$$
\begin{aligned}
\psi\left(\mathbf{s}_{i}, \mathbf{p}_{j}\right)=\gamma_{\mathbf{s}_{i}}^{-1} & \sum_{n=1}^{\infty} c_{n} f^{n-1} \mathbf{m}_{j} . \\
& {\left[\mathbf{r}_{j 0} P_{n}(\cos (\zeta))+\mathbf{t}_{j 0} P_{n}^{1}(\cos (\zeta))\right], }
\end{aligned}
$$

where $\gamma_{\mathbf{s}}=4 \pi \sigma_{3}\left\|\mathbf{s}_{i}\right\|, f=\frac{\left\|\mathbf{r}_{j}\right\|}{\left\|\mathbf{s}_{i}\right\|}$ and $\mathbf{p}_{j}$ is the parameter vector of dipole $j$ comprised of $\mathbf{r}_{j}$ and $\mathbf{m}_{j}$, i.e. $\mathbf{p}_{j}=\left\{\mathbf{r}_{j}, \mathbf{m}_{j}\right\}$. The radial and tangential unit vectors $\mathbf{r}_{j 0}$ and $\mathbf{t}_{j 0}$ depend on $\mathbf{r}_{j}$ and $\mathbf{s}_{i} . P_{n}($.$) and P_{n}^{1}($.$) denote the Legendre and the as-$ sociated Legendre polynomial of degree n, also $\zeta$ is the angle between $\mathbf{r}_{j}$ and $\mathbf{s}_{i}$. Also $c_{n}=\frac{(2 n+1)^{4}}{\Gamma}$ where:

$$
\begin{aligned}
\Gamma & =(2 n+1)\left(\frac{\sigma_{1}}{\sigma_{2}} n+n+1\right)\left[\left(\frac{\sigma_{2}}{\sigma_{3}} n+n+1\right)+(n+1)\left(\frac{\sigma_{2}}{\sigma_{3}}-1\right)\left(\frac{R_{1}}{R_{3}}\right)^{2 n+1}\right] \\
& +(n+1) \frac{R_{1}}{R_{2}}\left[(2 n+1)\left(\frac{\sigma_{1}}{\sigma_{2}}\right)\right]\left[n\left(\frac{\sigma_{2}}{\sigma_{3}}-1\right)+\left(\frac{\sigma_{2}}{\sigma_{3}} n+\frac{\sigma_{2}}{\sigma_{3}}+n\right)\left(\frac{R_{1}}{R_{3}}\right)^{2 n+1}\right] .
\end{aligned}
$$

$R_{1}, R_{2}, R_{3}$ are the radius of the spheres from interior to exterior and $\sigma_{1}, \sigma_{2}, \sigma_{3}$ are the corresponding conductivity values of the regions defined by these radiuses. So, the measured ERP signal at the $i^{\text {th }}$ electrode location can be expressed as:

$$
C h_{i}(t)=\sum_{j=1}^{N_{s}} a_{j}(t) \psi\left(\mathbf{s}_{i}, \mathbf{p}_{j}\right)+n_{i}(t),
$$

where $n_{i}(t)$ is additive noise, typically modeled as Gaussian white noise that is also uncorrelated between channels.

\section{OPTIMAL ERP SOURCE RECONSTRUCTION BY USING PARTICLE SWARM OPTIMIZATION}

In our proposed dipole source reconstruction technique, we choose the dipole source parameters to minimize the follow- ing cost function:

$$
J=\sum_{i=1}^{N_{c}}\left\|C h_{i}(t)-\tilde{C} h_{i}(t)\right\|^{2},
$$

which is a function of the following dipole source parameters: $\left\{a_{j}(t), \mathbf{p}_{j}\right\}, j=1,2, . ., N_{s}$. Here, $C h_{i}(t)$ is the available $N_{c}$ number of ERP channels, and $\tilde{C} h_{i}(t)$ is the forward solution for the potential measured at electrode $i$ for a given set of dipole parameters: $\left\{\tilde{a}_{j}(t), \tilde{\mathbf{p}}_{j}\right\}$ for $N_{s}$ number of dipoles. Since, as shown below, once the dipole positions and their orientations are specified, the optimal $a_{j}(t)$ 's can be found easily by a least squares solution, the optimization can be carried over the dipole position and orientation. This implies that, the optimization can be performed by a global search technique in a $5 N_{s}$ dimensional space for $N_{s}$ number of dipole sources. To show that given the dipole positions and orientations, their corresponding $a_{j}(t)$ 's can be determined by a least squares solution, we introduce the following matrix formulation:

$$
\mathbf{C h}_{k}=\boldsymbol{\Psi} \mathbf{A}_{k}+\mathbf{n}_{k}
$$

where $\mathbf{C h}_{k}=\left[C h_{1}\left(t_{k}\right), C h_{2}\left(t_{k}\right), . ., C h_{N c}\left(t_{k}\right)\right]^{T}, \mathbf{n}_{k}$ is the noise vector, $\Psi(i, j)$ is $\psi\left(\mathbf{s}_{i}, \mathbf{p}_{j}\right)$ in (2) and $\mathbf{A}_{k}=$ $\left[a_{1}\left(t_{k}\right), a_{1}\left(t_{k}\right), . ., a_{N c}\left(t_{k}\right)\right]^{T}$. Therefore, given dipole positions and orientations, the optimal value of $\mathbf{A}_{k}$ that minimizes the least squares fit error $\left\|\mathbf{C h}_{k}-\mathbf{\Psi} \mathbf{A}_{k}\right\|^{2}$ can be found as:

$$
\tilde{\mathbf{A}}_{k}=\left(\boldsymbol{\Psi}^{T} \boldsymbol{\Psi}\right)^{-1} \boldsymbol{\Psi}^{T} \mathbf{C h}_{k} .
$$

By using the optimal solution for the $\mathbf{A}_{k}$, the required optimization which is reduced to a search on the dipole positions and their orientations can be restated as:

$$
\left\{\tilde{\mathbf{p}}_{1}, \tilde{\mathbf{p}}_{2} \ldots \tilde{\mathbf{p}}_{N s}\right\}=\underset{\left\{\mathbf{p}_{1}, \mathbf{p}_{2}, \ldots, \mathbf{p}_{N s}\right\}}{\arg \min } \sum_{k}\left\|\mathbf{e}\left(t_{k}\right)\right\|^{2},
$$

where, $\mathbf{e}\left(t_{k}\right)$ 's are defined as:

$$
\mathbf{e}\left(t_{k}\right)=\mathbf{C h}_{t_{k}}-\boldsymbol{\Psi} \tilde{\mathbf{A}}_{t_{k}}=\left[\mathbf{I}-\boldsymbol{\Psi}\left(\boldsymbol{\Psi}^{T} \boldsymbol{\Psi}\right)^{-1} \boldsymbol{\Psi}^{T}\right] \mathbf{C h}_{t_{k}} .
$$

This expression can be simplified further as:

$$
\left\|\mathbf{e}\left(t_{k}\right)\right\|^{2}=\mathbf{C h}_{k}^{T} \mathbf{P} \mathbf{C h}_{k},
$$

where $\mathbf{P}=\mathbf{I}-\boldsymbol{\Psi}\left(\boldsymbol{\Psi}^{T} \boldsymbol{\Psi}\right)^{-1} \boldsymbol{\Psi}^{T}$ is a projection matrix. Although, the search dimension is greatly reduced in this form of the optimization, it is still $5 \times N_{s}$ dimensional with potentially multiple local and global minimas. Hence, gradient decent type local minimization techniques fails to provide reliable solutions. Therefore, a global optimization technique with fast convergence should be used. For this purpose we implemented the Particle Swarm Optimization technique [9]. As is well known, this powerful optimization technique makes use of particle $\mathrm{x}$ and includes a simple particle location update equation (9). In our application, particle 
$x=\left[r_{x}, r_{y}, r_{z}, \theta, \phi\right]^{T}$ where $r_{x}, r_{y}, r_{z}, \theta, \phi$ are the locations and the orientations of a dipole source. We selected the parameters of this update equation as $\chi=0.72984$, $c_{1}=c_{2}=2.05$, exactly same as suggested in [10].

$$
\vec{v}_{i d}=\chi\left(v_{i d}+c_{1} \epsilon_{1}\left(p_{i d}-x_{i d}\right)+c_{2} \epsilon_{2}\left(p_{g d}+x_{i d}\right)\right),
$$

where $x_{i d}$ is a possible solution defined by the $i^{\text {th }}$ particle location in the search space, $p_{i d}$ is its best solution and $p_{g d}$ is the global best solution among all particles until the current iteration. Solution space boundaries are chosen such that the estimated source locations remain inside the volume of the head. Here, it is assumed that $N_{s}$, the number of dipole sources is known. In practice typically the reconstruction techniques makes use of about 3 or 4 dipole sources. Therefore, we propose to gradually increase the number of sources used in the iterations, until the fit error energy is lowered to the level determined by the estimated noise level in the measured ERP channels. At the end of this cycle, the PSO provides optimal positions and the orientations of the dipoles: $\left\{\tilde{\mathbf{p}}_{1}, \tilde{\mathbf{p}}_{2} \ldots \tilde{\mathbf{p}}_{N s}\right\}$. Their corresponding dipole magnitude matrix $\mathbf{A}$, can be found using (6). Once all these parameters are determined, the forward model given in (1) can be used to obtain channel data corresponding to individual dipole sources, which are also valuable information for the investigation of the cognitive processes.

\section{RESULTS AND COMPARISONS}

To demonstrate the accuracy of proposed source reconstruction technique, first we will report its results on a 64 electrode synthetic ERP data which is generated by using the forward model (1) for 3 dipoles with known locations, orientations and time varying modulations. To the synthetically generated ERP channels, we added white noise so that the Signal to Noise Ratio (SNR) is about $15 \mathrm{~dB}$. In Fig.1a, timevarying modulation signals for three effective dipole sources are shown. Note that, as shown in Fig.1b, these synthetic signals are chosen such that they overlap both in time and frequency, making it very difficult to separate them in the raw channel data by using time-frequency filtering techniques. We started the optimization with a single dipole and then the program automatically increased the number of dipoles to two and finally to three. The average fit error results for each number of dipoles used in the optimization are shown in Fig. 1c. As seen from this figure, the average fit error decreases to the noise level as the number of dipoles increases from one to three. As given in Table 1, the position errors of the estimated dipoles are within $1 \mathrm{~mm}$. In Fig. 2a, the synthetic ERP signal at electrode 13, and its reconstruction based on the three dipole model is shown. As seen from this figure, the dipole reconstruction significantly reduces the noise in the recorded channel and closely matches the noise-free ERP signal. In addition, In Fig. 2b-2d, we show the ERP signal components at electrode 13 generated by the individual dipoles.
To illustrate the performance of the proposed source reconstruction technique on real data, we used 64 channel ERP recording in an oddball easy paradigm. We started our processing by first identifying the parameters of the best fitting sphere to the known electrode positions by solving the following minimization problem:

$$
\underset{\{\mathbf{c}, R\}}{\arg \min } \sum_{k=1}^{N_{c}}\left[\left\|\mathbf{r}_{k}-\mathbf{c}\right\|-R_{c}\right]^{2},
$$

where $\mathbf{r}_{k}=\left\{x_{k}, y_{k}, z_{k}\right\}$ are the coordinates of the $k^{\text {th }}$ electrode, $\mathbf{c}=\left\{x_{c}, y_{c}, z_{c}\right\}$ is the center of the sphere and $R_{c}$ is the sphere radius. These parameters of the best fitting sphere are used in the forward model. Like in the synthetic case, we started the optimization with a single dipole and then the program automatically increased the number of dipoles to two and to three. The average fit error results for each number of dipoles used in the optimization are shown in Fig. 3a. As seen from this figure, the average fit error decreases as the number of dipoles increases to three. Since with three dipoles the residual signal has reached to the noise level on the recorded data, the optimization is automatically terminated. To see the effect of using four dipoles, we manually restarted the optimization with four dipoles but could only get an insignificant improvement in the fit errors. In Fig. 3b, the real ERP signal at electrode 13 , and its reconstruction based on the three dipole model is shown. As seen from this figure, the dipole reconstruction significantly reduces the noise in the real ERP signals as well.

\section{CONCLUSIONS}

A new source reconstruction technique is proposed for accurate identification of ERP sources related with cognitive processes. In this new approach, positions, orientations and time-varying modulation parameters of all the dipoles are simultaneously optimized to obtain the least squares fit between the measured ERP channels and the synthetically generated ones based on a dipole source model. It is analytically shown that, the massive optimization problem can be reduced to a simpler one by eliminating the time-varying modulation parameters from the parameter search list. Then, the reduced optimization problem is solved by the Particle Swarm Optimization technique, which provides accurate solutions in a short duration of about 15 minutes on a computer with a Intel Xeon(R) 1.6GHz processor. By using synthetic and real ERP signals, the accurate performance of the proposed technique is illustrated. Most notably, even when the ERP sources generate signals that do overlap both in time and frequency, the proposed technique can identify their sources and separate the signals from one another. Hence, the proposed technique enables accurate topographic reconstruction of individual ERP components, potentially creating significant impact on the understanding of cognitive processes. 


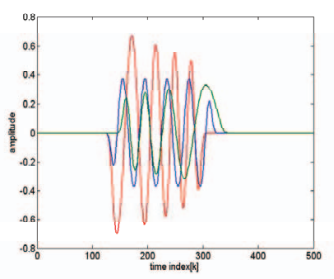

(a)

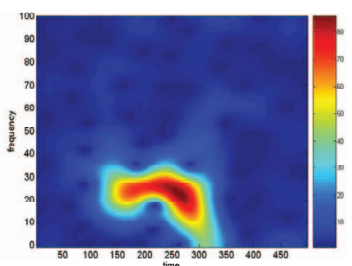

(b)

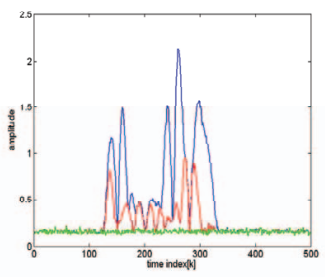

(c)
Fig. 1. (a) Modulation signals of the dipoles. (b) Timefrequency distribution of the $13^{\text {th }}$ channel. (c)The fit error for 1(blue), 2(red) and 3(green) dipole optimization. Note that the fit error with 3 dipoles is at the noise level.

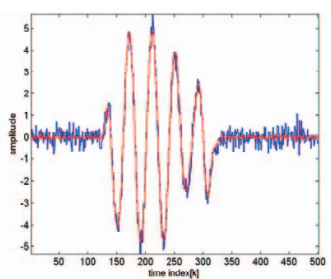

(a)

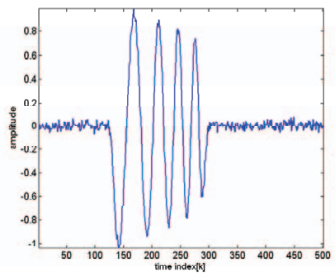

(c)

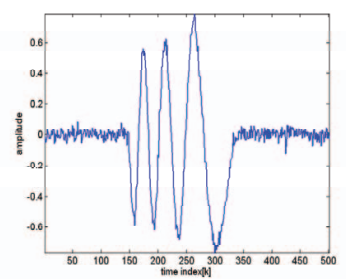

(b)

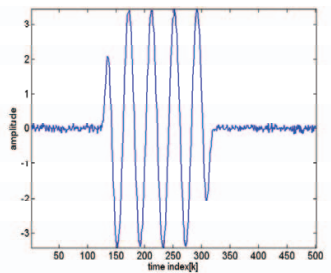

(d)
Fig. 2. (a)The measured(blue) and reconstructed(red) $13^{\text {th }}$ electrode signal. (b-d)Signal components generated by individual dipoles at the location of the $13^{\text {th }}$ electrode.

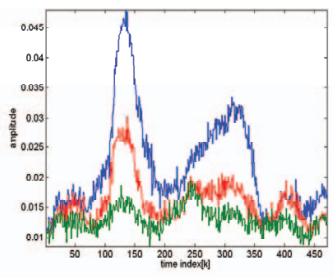

(a)

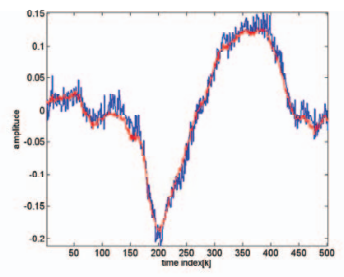

(b)
Fig. 3. (a)The fit error for 1(blue), 2(red) and 3(green) dipole optimization. (b) The measured(blue) and reconstructed(red) $13^{\text {th }}$ electrode signal.

\begin{tabular}{|c|c|c|c|c|c|c|}
\hline & $\mathbf{r}_{1}$ & $\tilde{\mathbf{r}}_{1}$ & $\mathbf{r}_{2}$ & $\tilde{\mathbf{r}}_{2}$ & $\mathbf{r}_{3}$ & $\tilde{\mathbf{r}}_{3}$ \\
$s_{x}(\mathrm{~mm})$ & -10 & -9.97 & -30 & -29.12 & 40 & 40.67 \\
\hline$s_{y}(\mathrm{~mm})$ & 31 & 31.33 & -10 & -9.76 & -21 & -21.34 \\
\hline$s_{z}(\mathrm{~mm})$ & 70 & 69.92 & 55 & 54.23 & 85 & 84.78 \\
\hline
\end{tabular}

Table 1. The original $\mathbf{r}_{k}$ and the reconstructed $\tilde{\mathbf{r}}_{k}$ dipole source coordinates for $\mathrm{k}^{\text {th }}$ dipole. The reconstruction error is smaller than 1 millimeter.

\section{REFERENCES}

[1] M. Liotti, S.R. Pliszka, R. Perez, D. Kothmann, and M.G. Woldorff, "Abnormal brain activity related to performance monitoring and error detection in children with adhd," Cortex, vol. 41, pp. 377-388, 2005.

[2] J.C. Mosher, P.S. Lewis, and R.M. Leahy, "Multiple dipole modeling and localization from spatio-temporal meg data," IEEE Trans. Biomed. Eng., vol. 39, pp. 541577, June 1992.

[3] Z.J. Koles, "Trends in eeg source localization," Electroenceph. clin. Neurophysiol, vol. 106, pp. 127-137, February 1998.

[4] R.M. Gulrajani, F.A. Roberge, and P. Savard, "Moving dipole inverse ecg and eeg solutions," IEEE Trans. Biomed. Eng., vol. 31, pp. 903-910, December 1984.

[5] H. Hallez, B. Vanrumste, R. Grech, J. Muscat, W.D. Clerq, A. Vergult, Y. Dasseler, K.P. Camilleri, S. G. Fabri, S.V. Huffel, and I. Lemahieu, "Review on solving the forward problem in eeg source analysis," Journal of NeuroEng. and Rehabil., vol. 4, pp. 46:75, 2007.

[6] R.D. Pascual-Marqui, "Standardized low resolution brain electromagnetic tomography (sloreta): technical details," Methods Find. Exp. Clin. Pharmacol., vol. 24, pp. 5-12, 2002.

[7] M. Sun, "An efficient algorithm for computing multishell spherical volume conductor models in eeg dipole source localization," IEEE Trans. Biomed. Eng., vol. 44, pp. 1243-1252, December 1997.

[8] S. Gabriel, R.W. Lau, and C. Gabriel, "The dielectric properties of biological tissues: Ii. measurements in the frequency range $10 \mathrm{hz}$ to $20 \mathrm{ghz}$," Phys. Med. Biol., vol. 41, pp. 2251-2269, 1996.

[9] J. Kennedy and R. Eberhart, "Particle swarm optimization," IEEE Int. Conf. Neural Networks, vol. 4, pp. 1942-1948, November 1995.

[10] D. Bratton and J. Kennedy, "Defining a standard for particle swarm optimization," IEEE Swarm Intelligence Symposium, pp. 120-127, April 2007. 